Article

\title{
Synthesis, Crystal Structure and Nonlinear Optical Property of $\mathrm{RbHgI}_{3}$
}

\author{
Yanjun Li ${ }^{1, *}$, Yuxun Ding ${ }^{1}$, Yaming Li ${ }^{1}$, Hongming Liu ${ }^{2}$, Xianggao Meng ${ }^{3}$, Ye Cong ${ }^{1}$, \\ Jiang Zhang ${ }^{1}$, Xuanke Li ${ }^{1}$, Xingguo Chen ${ }^{2}$ and Jingui Qin ${ }^{2, *}$ \\ 1 Department of Chemistry, Wuhan University of Science and Technology, Wuhan 430081, China; \\ dingyx0518@163.com (Y.D.); liyaming0806@163.com (Y.L.); congye626@126.com (Y.C.); \\ zhangjiang@wust.edu.cn (J.Z.); xkli@21cn.com (X.L.) \\ 2 Department of Chemistry, Wuhan University, Wuhan 430072, China; hongming9224@126.com (H.L.); \\ xgchen@whu.edu.cn (X.C.) \\ 3 College of Chemistry, Central China Normal University, Wuhan 430079, China; \\ mengxianggao@mail.ccnu.edu.cn \\ * Correspondence: yanwatercn@wust.edu.cn (Y.L.); jgqin@wdu.edu.cn (J.Q.); \\ Tel.: +86-027-8655-6906 (Y.L.); +86-027-6875-2330 (J.Q.)
}

Academic Editors: Ning Ye and Rukang Li

Received: 15 January 2017; Accepted: 16 May 2017; Published: 22 May 2017

\begin{abstract}
Searching for new nonlinear optical (NLO) crystals to be used in the infrared (IR) region is still a challenge. This paper presents the synthesis, crystal structure and properties of a new halide, $\mathrm{RbHgI}_{3}$. Its non-centrosymmetric single crystal can be grown in solution. In its crystal structure, all the polar $\left[\mathrm{HgI}_{4}\right]^{2-}$ groups align in such a way that brings a favorable net polarization. The measurement by Kurtz-Perry powder technique indicates that $\mathrm{RbHgI}_{3}$ shows a phase-matchable second harmonic generation (SHG) property seven times stronger than that of $\mathrm{KH}_{2} \mathrm{PO}_{4}(\mathrm{KDP})$. $\mathrm{RbHgI}_{3}$ displays excellent transparency in the range of $0.48-25 \mu \mathrm{m}$ with relatively good thermal stability. The UV absorption implies that this yellow compound's band gap is about $2.56 \mathrm{eV}$, close to that of $\mathrm{AgGaS}_{2}$. A preliminary measurement indicates that the laser-induced damage threshold of the crystal is about $28.3 \mathrm{MW} / \mathrm{cm}^{2}$. These preliminary experimental data reveal that $\mathrm{RbHgI}_{3}$ is a new candidate as nonlinear optical material in the infrared region.
\end{abstract}

Keywords: nonlinear optical crystal; infrared region; $\mathrm{RbHgI}_{3}$; synthesis; crystal structure

\section{Introduction}

Second-order nonlinear optical (NLO) crystals are very important high-tech materials due to their application in laser frequency convention, optical parameter oscillator (OPO), and signal communication [1,2]. According to wavelength range, second-order NLO materials can be classified into three categories, namely ultraviolet NLO crystals, visible NLO crystals and infrared NLO crystals. Great progress has been made in ultraviolet NLO crystals in the last several decades. For instance, some excellent new crystals such as $\beta-\mathrm{BaB}_{2} \mathrm{O}_{4}(\mathrm{BBO})$ [3], $\mathrm{LiB}_{3} \mathrm{O}_{5}$ (LBO) [4], $\mathrm{KBe}_{2} \mathrm{BO}_{3} \mathrm{~F}_{2}(\mathrm{KBBF})$ [5-7] have been developed by Chuangtian $\mathrm{Chen}$ and his colleagues. In the visible region, there are some excellent $\mathrm{NLO}$ crystals such as $\mathrm{KH}_{2} \mathrm{PO}_{4}$ (KDP) [8], $\mathrm{KTiOPO}_{4}$ (KTP) [9] and so forth. Nevertheless, numerous NLO crystals in IR regions are not fully satisfied. Some common infrared NLO crystals, such as $\mathrm{AgGaS}_{2}$ [10], $\mathrm{AgGaSe}_{2}$ [11] and $\mathrm{ZnGeP}_{2}$ [12] possess two drawbacks: one is their low laser damage thresholds (LDT), and the other is not easy to grow high-quality crystals. Therefore, searching for new infrared NLO crystals is still a challenge in this field.

However, general knowledge indicates that high LDT of the materials originates from the large gap. Guided by this purpose, thousands of IR NLO crystals have been discovered and many materials 
exhibit excellent performance such as halides, chalcogenides, iodates and phosphides etc., Two review articles $[13,14]$ about IR NLO materials have been recently published. It has been accepted that the band gap which causes thermal and electronic effects is in accord with laser damage threshold $[15,16]$. As we know, halides usually exhibit large band gaps, and there are four halogen atoms to choose from. Moreover, halides can be easily dissolved in solvents so that it is easier to grow single crystals. On the other hand, mercury atom is a heavy metal element. This is beneficial for the wide transparence in the IR region. Furthermore, the mercury (II) cation $\left(\mathrm{Hg}^{2+}\right)$ possesses $\mathrm{d}^{10}$ electronic configuration and does not emerge $d-d$ transition. This will lead to relatively wide band gaps. Therefore, with Chuangtian Chen's long-term help and collaboration, we have pursued research on exploring new second-order NLO crystals to be used in the mid-IR region from halides, and some new potential mid-IR NLO crystals such as $\mathrm{Cs}_{2} \mathrm{HgCl}_{2} \mathrm{I}_{2}$ [17], $\mathrm{Cs}_{2} \mathrm{Hg}_{3} \mathrm{I}_{8}$ [18], $\mathrm{HgBrI}$ [19], $\mathrm{HgBr}_{2}$ [20], $\mathrm{Hg}_{2} \mathrm{BrI}_{3}$ [21], $\mathrm{CsHgBr}_{3}$ [22], $\beta-\mathrm{HgBrCl}$ [23], $\mathrm{NaSb}_{3} \mathrm{~F}_{10}$ [24] and $\mathrm{Rb}_{2} \mathrm{CdBr}_{2} \mathrm{I}_{2}$ [25] have been developed by this idea on our group.

In this paper, we report the synthesis, crystal structure, and NLO properties of a new compound $\mathrm{RbHgI}_{3}$. The compound is pure $\mathrm{RbHgI}_{3}$ without crystalline water and it differs from the compound of $\mathrm{RbHgI}_{3} \cdot \mathrm{H}_{2} \mathrm{O}$ reported in the literature [26]. The powder second harmonic generation (SHG) effect of $\mathrm{RbHgI}_{3} \cdot \mathrm{H}_{2} \mathrm{O}$ is very weak and not phase-matchable. The existence of the crystalline water is harmful for thermal stability and transparent region in the IR region. Differently, $\mathrm{RbHgI}_{3}$ shows a powder second harmonic generation (SHG) property seven times as high as that of KDP. It also exhibits a wide transparent window in the infrared region. It also shows reasonable band gap and thermal stability. To conclude, $\mathrm{RbHgI}_{3}$ is a potential candidate as new IR NLO material.

\section{Results and Discussion}

\subsection{Synthesis and Analysis}

$\mathrm{RbHgI}_{3}$ was synthesized with $\mathrm{HgI}_{2}$ and $\mathrm{RbI}$ by conventional solution reaction. The phase purity was checked by powder XRD. No impurities were observed, and the diffraction patterns are not consistent with those of $\mathrm{HgI}_{2}$ [27] or RbI [28] phase, and also not the sum of $\mathrm{HgI}_{2}$ and $\mathrm{RbI}$ phases by the XRD contrast analyses. The measured powder X-ray diffraction patterns are in agreement with the simulated patterns based on the single crystal structure of $\mathrm{RbHgI}_{3}$ obtained from the solution reaction (See Figure 1).

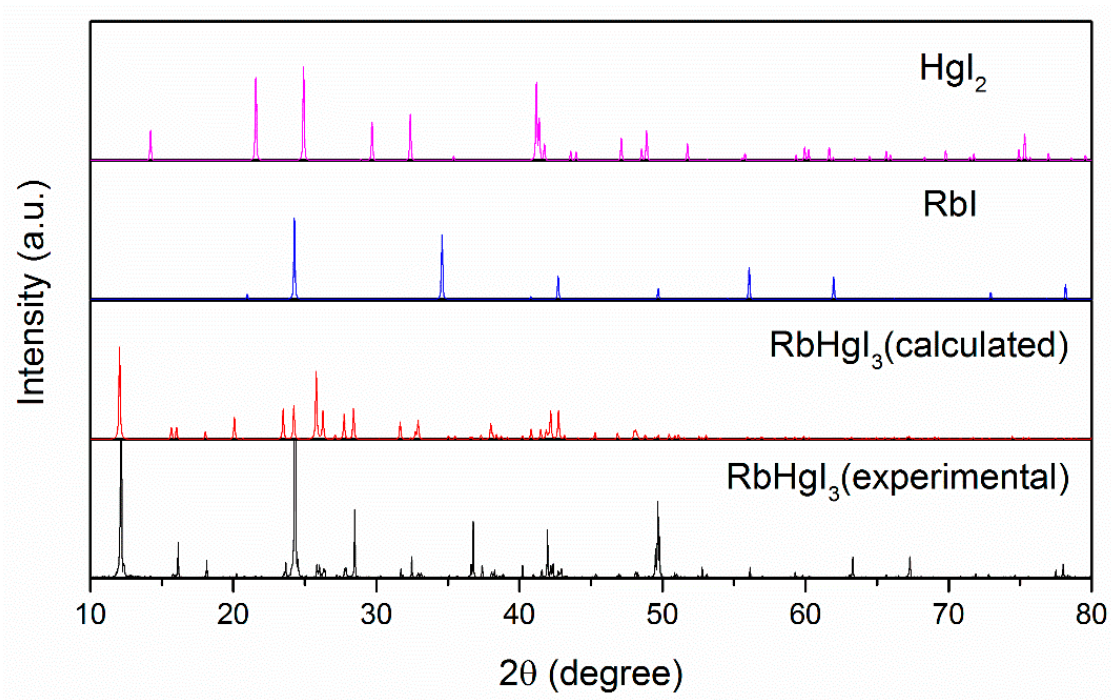

Figure 1. Comparison of experimental and calculated powder X-ray diffraction patterns: the experimental powder $\mathrm{X}$-ray diffraction data of $\mathrm{RbHgI}_{3}$ powders obtained from the solution reaction; the calculated powder X-ray diffraction data of $\mathrm{HgI}_{2}, \mathrm{RbI}$ and $\mathrm{RbHgI}_{3}$ from single crystal structure data. 


\subsection{Crystal Structure}

$\mathrm{RbHgI}_{3}$ crystallizes in the noncentrosymmetric orthorhombic space group Ama2 (No. 40). Figure 2a shows the packing diagram of $\mathrm{RbHgI}_{3}$ along $b c$ plane. Each $\left[\mathrm{HgI}_{4}\right]^{2-}$ group forms a distorted tetrahedron (Figure $2 b$ ), which is connected with the neighbors by sharing an iodine atom to form a one-dimensional (1D) zigzag chain along the $a$-axis (Figure 2c). The chains are then to form layers along the $a b$ plane, and the planes are further connected into a three-dimensional (3D) framework by the $\mathrm{Rb}$ atoms that occupy the empty spaces surrounded by iodine atoms (Figure 2). In the crystal structure, it also exhibits disorder of $\mathrm{Rb}$ atom in the symmetry unit with $50 \%$ occupancies (Figure 2a). As shown in Figure $2 \mathrm{~b}$, in each $\left[\mathrm{HgI}_{4}\right]^{2-}$ group there are two types of $\mathrm{Hg}$-I bond lengths, namely,

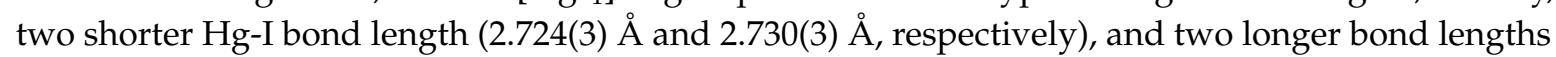
(both 2.868(16) $\AA$ ). Interestingly, all the shorter $\mathrm{Hg}$-I bonds are always located above the $\mathrm{Hg}$ atoms in each $\left[\mathrm{HgI}_{4}\right]$ tetrahedron (Figure 2c). This packing style gives rise to a net polarization parallel to the direction of the $c$ axis (indicated by the black arrow in Figure 2b,c). Due to the favorable alignment and high density of the $\left[\mathrm{HgI}_{4}\right]^{2-}$ anionic group, the compound can show a relatively strong SHG response.

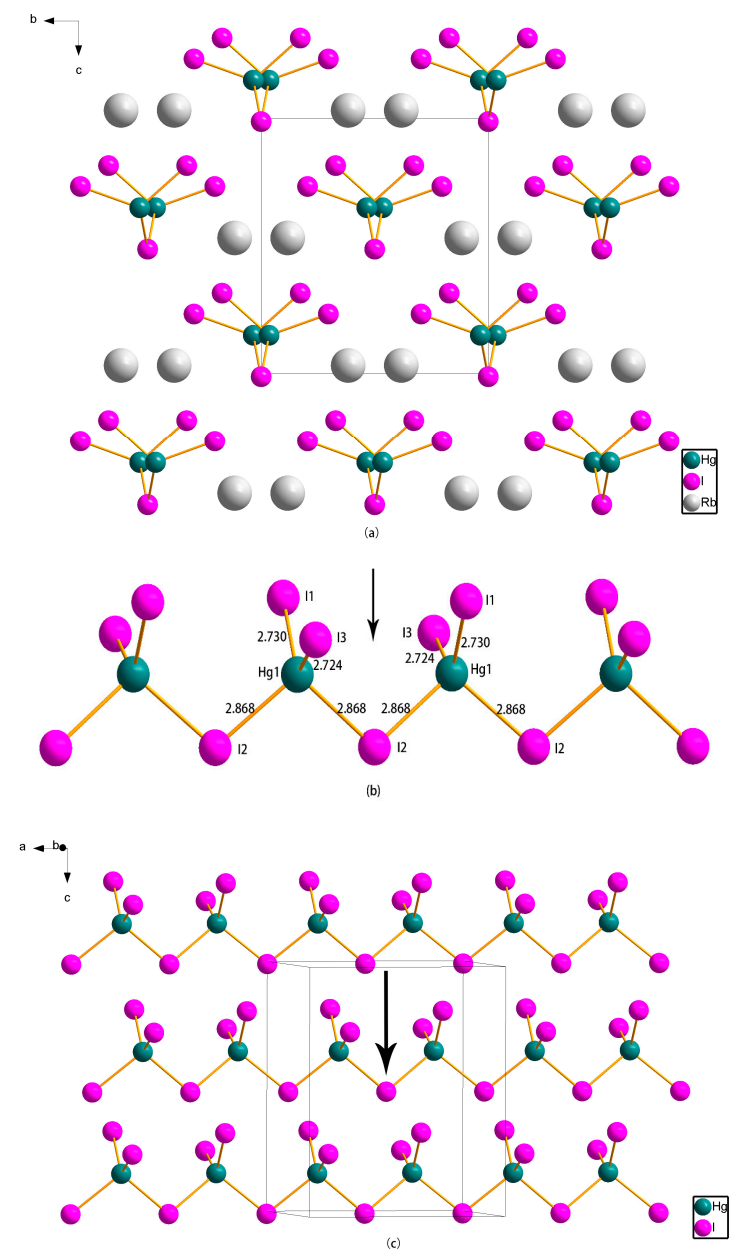

Figure 2. (a) Ball-and-stick packing diagrams of $\mathrm{RbHgI}_{3}$ along bc plane; (b) $\left[\mathrm{HgI}_{4}\right]$ tetrahedron in a unit cell. Each $\mathrm{Hg}$ atom is bonded to four iodine atoms with two types of the bond length, namely, two are short (2.730(3) and 2.724(3) $\AA$ ) and the other two are longer (2.868(16) $\mathrm{A})$; (c) Ball-and-stick diagrams of $\left[\mathrm{HgI}_{4}\right]$ tetrahedron ( $\mathrm{Rb}$ atoms are omitted for clarity). All the longer $\mathrm{Hg}$-I bonds are always located below the $\mathrm{Hg}$ atom in each $\left[\mathrm{HgI}_{4}\right]$ tetrahedron, giving rise to a net dipole moment parallel to the $\mathrm{c}$ axis (indicated by the black arrow). 


\subsection{Infrared Spectrum and $U V$-Vis Diffuse Reflectance Spectrum}

The attenuated total reflection Fourier-transformed infrared (ATR-FTIR) spectrum of $\mathrm{RbHgI}_{3}$ crystalline samples is shown in Figure 3. The ATR-FTIR spectrum indicates no absorption in the middle IR region from 4000 to $400 \mathrm{~cm}^{-1}(2.5-25 \mu \mathrm{m})$. This agrees quite well with the reported compounds such as $\mathrm{Tl}_{4} \mathrm{HgI}_{6}$ [29] and $\mathrm{Cs}_{2} \mathrm{Hg}_{3} \mathrm{I}_{8}$ [18,30], and they are transparent between 4000 and $400 \mathrm{~cm}^{-1}$. According to the references, $\mathrm{Tl}_{4} \mathrm{HgI}_{6}$ exhibits a broad range of transparency from 1.4 to $40 \mu \mathrm{m}$, while $\mathrm{Cs}_{2} \mathrm{Hg}_{3} \mathrm{I}_{8}$ is reported to be transparent between 0.5 and $25 \mu \mathrm{m}$. The UV-Vis diffuse reflectance spectrum of $\mathrm{RbHgI}_{3}$ is shown in Figure 4. The compound is yellow and the spectrum shows that the absorption edge near the UV side is about $484 \mathrm{~nm}$, and this indicates that the optical band gap of the compound is approximately $2.56 \mathrm{eV}$. Based on these data, the transparent range of $\mathrm{RbHgI}_{3}$ is $0.48-25 \mu \mathrm{m}$.

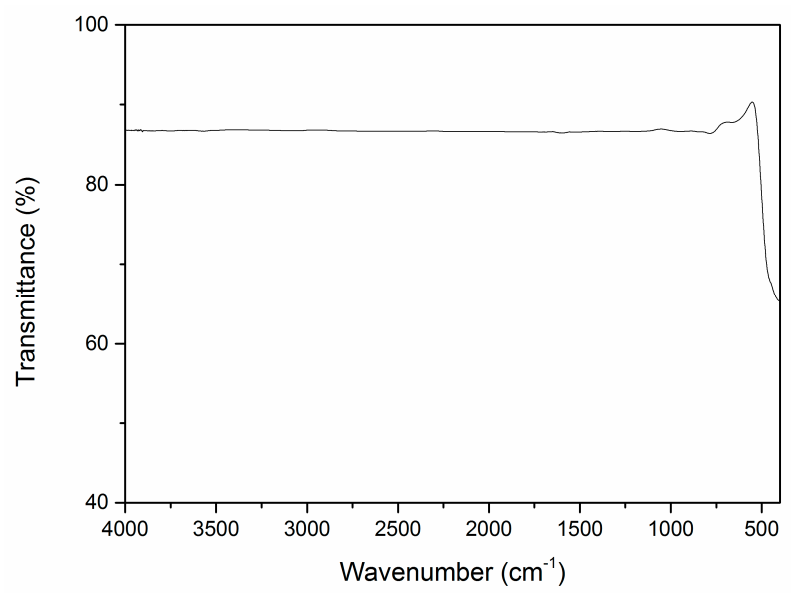

Figure 3. Attenuated total reflection Fourier-transformed infrared (ATR-FTIR) spectrum for $\mathrm{RbHgI}_{3}$.

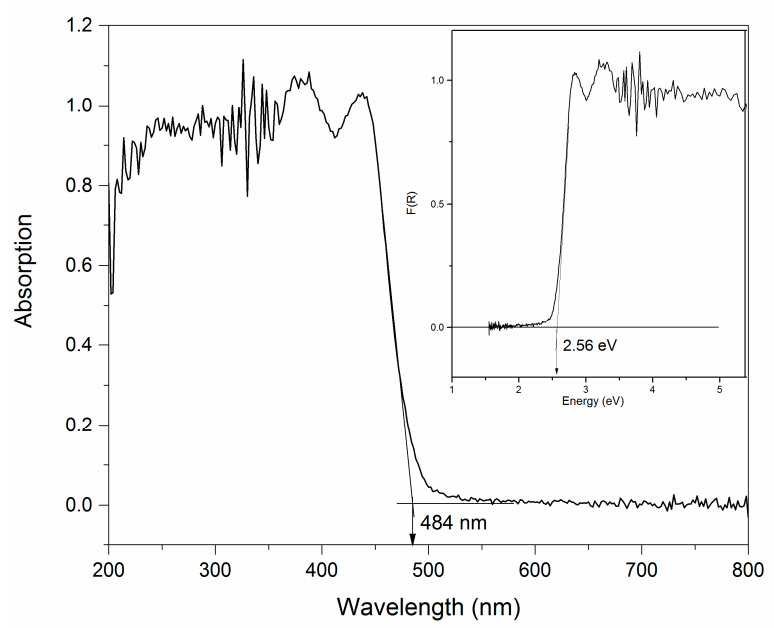

Figure 4. UV-Vis diffuse reflectance spectroscopy data for $\mathrm{RbHgI}_{3}$.

\subsection{NLO Property and LDT Measurement}

Powder SHG measurements using $1064 \mathrm{~nm}$ laser radiation revealed that $\mathrm{RbHgI}_{3}$ showed powder SHG efficiencies seven times as strong as that of KDP. The study result of the SHG intensity as a function of particle size (from 20 to $300 \mu \mathrm{m}$ ) is shown in Figure 5. The intensity of SHG signals at first increases gradually with the increase of the sample size and then reaches saturation when the sample size increases further. It is a typical curve for indicating that the $\mathrm{SHG}$ effect of $\mathrm{RbHgI}_{3}$ is phase-matchable [31]. 


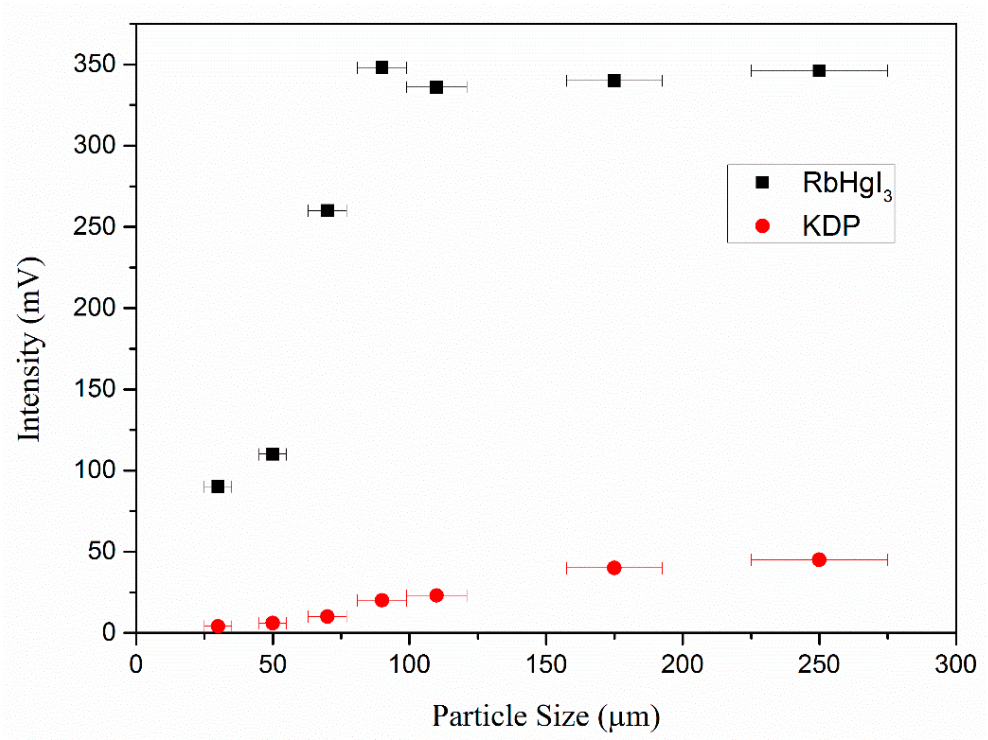

Figure 5. Second harmonic generation (SHG) intensity versus particle size for $\mathrm{RbHgI}_{3}$ and $\mathrm{KH}_{2} \mathrm{PO}_{4}$ (KDP).

A preliminary examination of the LDT measurement has been carried out on the crystalline sample using a Q-switched laser source. The samples showed a damage threshold of about $28.3 \mathrm{MW} / \mathrm{cm}^{2}$ $(1064 \mathrm{~nm}, 10 \mathrm{~ns})$, which is approximately equal to that of $\mathrm{AgGaS}_{2}\left(30 \mathrm{MW} / \mathrm{cm}^{2}, 1064 \mathrm{~nm}\right)$ [32].

\subsection{Thermogravimetric Analysis}

The yellow powder crystal of $\mathrm{RbHgI}_{3}$ is not hygroscopic and does not change its color after being exposed to air for about six months. The thermal behavior of $\mathrm{RbHgI}_{3}$ was investigated using thermogravimetric analysis (TGA). The TG curve reveals that the compound starts losing its weight when it is heated to above $120^{\circ} \mathrm{C}$ (See Figure 6), and then it continues to lose weight and reach a platform at about $450{ }^{\circ} \mathrm{C}$. The thermal stability of $\mathrm{RbHgI}_{3}$ is not very high and may be due to the existence of the iodine atom.

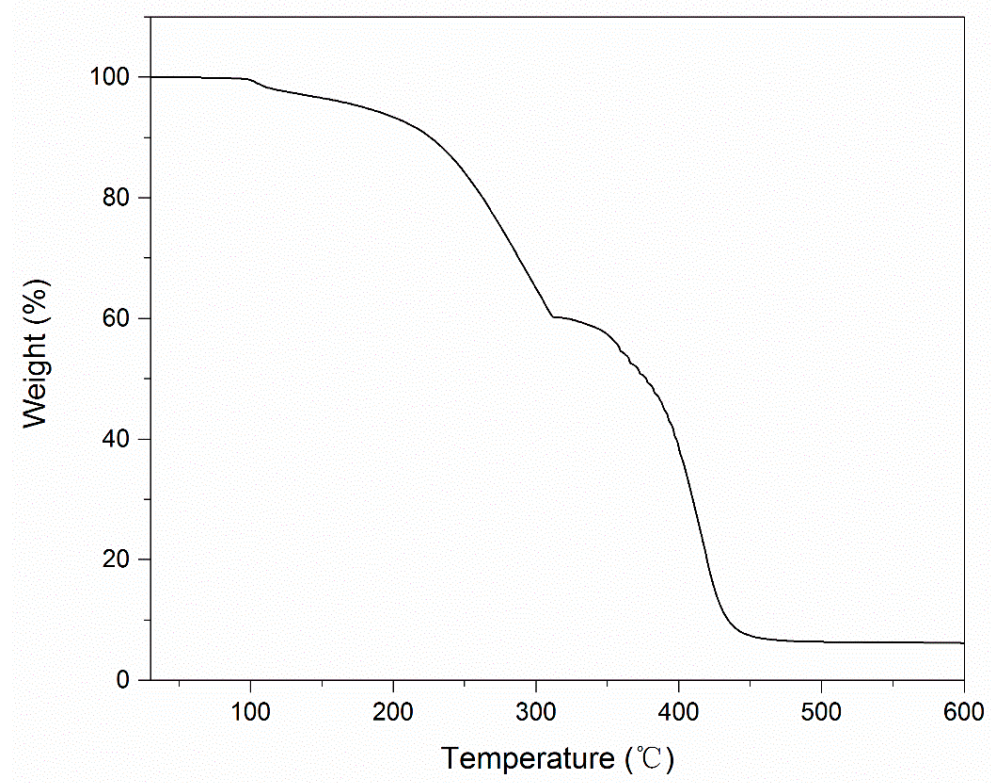

Figure 6. Thermogravimetric analysis (TGA) curve for $\mathrm{RbHgI}_{3}$. 


\subsection{Electronic Structure and Optical Properties Calculation}

To better understand the relationship of structure and the optical properties of the $\mathrm{RbHgI}_{3}$, the first-principles density functional theory (DFT) calculations were performed. The calculated band gap is $1.95 \mathrm{eV}$. The deviations from the experimental values should be attributed to the limitation of DFT [33]. Hence, scissors of $0.61 \mathrm{eV}$ has been added to shift up the conduction band (CB) levels in order to agree with the measured value of the band gap for the following calculations and analyses.

The partial density of states (PDOS) projected on the constitutional atoms in $\mathrm{RbHgI}_{3}$ are shown in Figure 7. The $\mathrm{Rb} s, \mathrm{Rb} 4 p, \mathrm{I} 5 s$ and $\mathrm{Hg} 5 d$ orbitals are strongly localized in the valence band (VB) about $-25 \mathrm{eV},-10 \mathrm{eV}$ and $-5.8 \mathrm{eV}$, respectively. The upper of the valence states from $-2.5 \mathrm{eV}$ show a large hybridization between $\mathrm{Hg} 5 p$ (and $5 d$ ) and I $5 p$ orbitals, indicating very strong chemical bonds between the $\mathrm{Hg}$ and I atoms, but the VB maximum is dominated by I $5 p$ orbitals which are higher than $\mathrm{Hg} 5 d$ orbitals in the VB. The bottom of CB is mainly composed of the $5 s$ and $5 p$ orbitals of I atom and $6 s$ orbitals of $\mathrm{Hg}$ atom, but I $5 s$ orbitals are lower than $\mathrm{Hg} 5$ s orbitals in the CB. This means that the iodide anion directly determines the energy band gap of $\mathrm{RbHgI}_{3}$. This is the reason why the laser damage threshold is not high and it agrees with the experimental value.

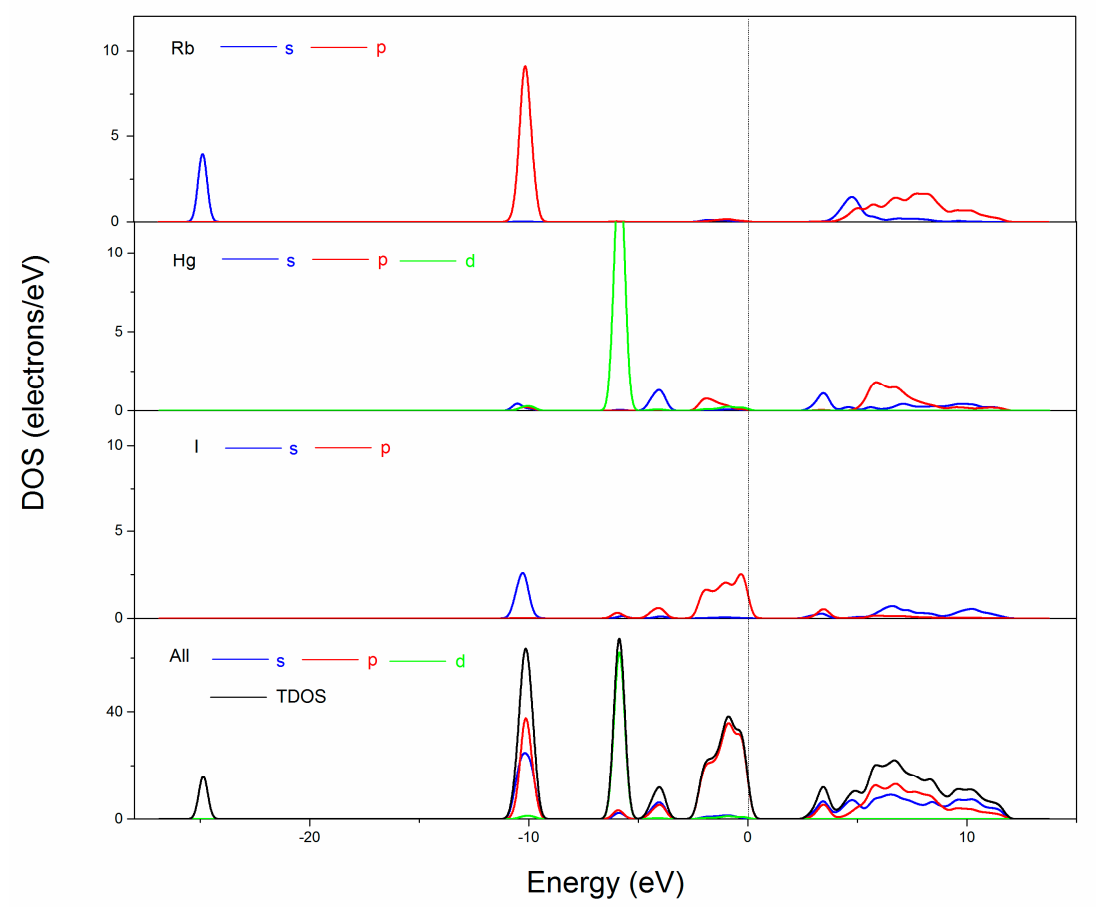

Figure 7. The partial density of states (PDOS) for $\mathrm{RbHgI}_{3}$.

On the basis of the above electronic band structure, the refractive indices and second harmonic generation (SHG) coefficients of $\mathrm{RbHgI}_{3}$ were obtained. The refractive indices are shown in Figure S2 (see the supporting information) and the calculated refractive indices and birefringence at several radiation wavelengths are listed in Table 1 . It is shown that the birefringence $\Delta n$ is larger than 0.08 as the wavelength is longer than $1000 \mathrm{~nm}$, so $\mathrm{RbHgI}_{3}$ is phase-matchable for the SHG in the IR region. Furthermore, we theoretically determined the SHG coefficients of $\mathrm{RbHgI}_{3}$. For the orthorhombic $m m 2$ point group and considering the restriction of Kleinman's symmetry, the title compound (Ama2) has three $\left(d_{15}, d_{24}\right.$ and $\left.d_{33}\right)$ independent SHG tensors and the static SHG coefficients are as follows: $d_{15}=28.99 \mathrm{pm} / \mathrm{V}, d_{24}=-27.19 \mathrm{pm} / \mathrm{V}$ and $d_{33}=2.95 \mathrm{pm} / \mathrm{V}$. The calculated results agree with the experimental observation that $\mathrm{RbHgI}_{3}$ exhibits a SHG response seven times that of $\mathrm{KDP}\left(d_{36}=0.39 \mathrm{pm} / \mathrm{V}\right)$. Therefore, we are confident that $\mathrm{RbHgI}_{3}$ possess strong $\mathrm{SHG}$ effects and is a promising candidate for the nonlinear optical applications. 
Table 1. Calculated Refractive Indices at Selected Wavelengths for $\mathrm{RbHgI}_{3}$.

\begin{tabular}{ccccc}
\hline Wavelength & $\boldsymbol{n}_{\mathbf{x}}$ & $\boldsymbol{n}_{\mathbf{y}}$ & $\boldsymbol{n}_{\mathbf{z}}$ & $\boldsymbol{\Delta} \boldsymbol{n}\left(\boldsymbol{n}_{\mathrm{y}}-\boldsymbol{n}_{\mathbf{x}}\right)$ \\
\hline $1000 \mathrm{~nm}$ & 2.01 & 2.09 & 2.07 & 0.08 \\
$1064 \mathrm{~nm}$ & 2.00 & 2.08 & 2.06 & 0.08 \\
$2000 \mathrm{~nm}$ & 1.97 & 2.05 & 2.03 & 0.08 \\
$\sim \infty$ & 1.96 & 2.04 & 2.02 & 0.08 \\
\hline
\end{tabular}

\section{Materials and Methods}

\subsection{Synthesis and Crystal Growth}

All the starting materials are analytically pure from commercial sources and used without further purification. $\mathrm{RbHgI}_{3}$ was synthesized by conventional solution reaction. $\mathrm{HgI}_{2}$ and $\mathrm{RbI}$ (mole ratio 1:1) are carefully dissolved in ethanol. The mixture was stirred at $80^{\circ} \mathrm{C}$ for $5 \mathrm{~h}$. The yellow solution was filtered and slowly cooled, then kept in a flask at room temperature. After a few days, some yellow crystals appeared in the bottom of the flask. The yellow crystals were filtered and carefully washed with cool ethanol. A single phase of $\mathrm{RbHgI}_{3}$ crystals was obtained with the yield of $65 \%$ (based on $\mathrm{HgI}_{2}$ ). The $\mathrm{RbHgI}_{3}$ crystal can be grown from the ethanol solution and a photograph of the $\mathrm{RbHgI}_{3}$ crystal is presented in Figure S1 (see the supporting information).

\subsection{Structure Determination}

A single crystal of $\mathrm{RbHgI}_{3}$ with dimensions of ca. $0.10 \times 0.10 \times 0.08 \mathrm{~mm}^{3}$ was selected and used for single-crystal diffraction experiment. Data sets were collected using a Bruker SMART APEX diffractometer (Bruker, Karlsruhe, Germany) equipped with a CCD detector (graphite-monochromated Mo-K $\alpha$ radiation $\lambda=0.71073 \AA$ ) at $298(2) \mathrm{K}$. Data sets reduction and integration were performed using the software package SAINT PLUS [34]. The crystal structure is solved by direct methods and refined using the SHELXTL 97 software package $[35,36]$. Single crystal data collection, cell parameters and basic information for $\mathrm{RbHgI}_{3}$ are summarized in Table 2. Further details of the crystal structure studies may be obtained from the Fachinformationszentrum Karlsruhe, 76344 Eggenstein-Leopoldshafen, Germany (Fax: (49) 7247808666; e-mail: crysdata@fiz-karlsruhe.de), on quoting registry number CSD-432180 for $\mathrm{RbHgI}_{3}$.

Table 2. Crystallographic data of $\mathrm{RbHgI}_{3}$.

\begin{tabular}{cc}
\hline Empirical Formula & RbHgI $_{3}$ \\
\hline Formula weight & 666.76 \\
Temperature & $296(2)$ \\
Wavelength & 0.71073 \\
Crystal color & Yellow \\
Crystal system & Orthorhombic \\
Space group & Ama2 $($ No.40) \\
Crystal size $\left(\mathrm{mm}^{3}\right)$ & $0.10 \times 0.10 \times 0.08$ \\
Unit cell dimensions $(\AA)$ & $0.838(3), b=9.824(3), c=11.050(3)$ \\
$Z$ & 4 \\
$V\left(\AA^{3}\right)$ & $959.4(5)$ \\
Absorption coefficient $\left(\mathrm{mm}^{-1}\right)$ & 30.640 \\
Density $($ calculated $)$ & 4.616 \\
Goodness-of-fit on $F^{2}$ & 1.149 \\
Reflections collected & 1473 \\
Independent reflection & $1337[\mathrm{R}(\mathrm{int})=0.0293]$ \\
$R_{1}, \mathrm{w} R_{1}[I>2 \sigma(I)]$ & $0.0682 / 0.1845$ \\
$R_{2}, \mathrm{w} R_{2}($ all data $)$ & $0.0637 / 0.1818$ \\
Min $/ \mathrm{max}^{2} \rho / \mathrm{e} \cdot \AA^{-3}$ & $-3.472 / 3.022$ \\
\hline$\omega=1 /\left[\mathrm{s}^{2}\left(\mathrm{Fo}^{2}\right)+(0.0702 \mathrm{P})^{2}+51.3875 \mathrm{P}\right]$, where $\mathrm{P}=\left(\mathrm{Fo}^{2}+2 \mathrm{Fc}^{2}\right) / 3$.
\end{tabular}




\subsection{Powder XRD Measurement}

X-ray powder diffraction (XRD) patterns of the polycrystalline material were collected using a Bruker D8 Advanced diffractometer (Bruker, Karlsruhe, Germany) with $\mathrm{Cu} k \alpha 1$ radiation $(\lambda=154186 \AA)$ in the range of $10-80^{\circ}(2 \theta)$ at a scanning rate of $6^{\circ} / \mathrm{min}^{-1}$.

\subsection{Optical Spectroscopy}

The optical transmission in the mid-IR region was recorded on a NICOLET 5700 Fourier-transformed infrared (FT-IR) spectrophotometer (Manufacture, City, Country) in the $4000-400 \mathrm{~cm}^{-1}(2.5-25 \mu \mathrm{m})$ region using the attenuated total reflection (ATR) technique with a diamond crystal. The crystal sample was loaded on the samples stage and then the ATR-FTIR spectrum was measured. The UV-Vis absorption spectrum was recorded on a Varian Cary 5000 UV-Vis-NIR spectrophotometer (Agilent, Palo Alto, CA, USA) in the region $200-800 \mathrm{~nm}$. A BaSO 4 plate was used as the standard (100\% reflectance), on which the finely ground samples from the crystals were coated. The absorption spectrum was calculated from the reflectance spectrum using the Kubelka-Munk [37] function: $\alpha / S=\left(1-R^{2}\right) /(2 R)$, where $\alpha$ is the absorption coefficient, $S$ is the scattering coefficient, and $\mathrm{R}$ is reflectance.

\subsection{Second-Harmonic Generation (SHG) and Laser Damage Threshold (LDT) Measurement}

The NLO efficiencies of the samples were investigated using a Kurtz-Perry powder technique [30]. A pulsed Q-switched Nd:YAG laser was utilized to generate fundamental $1064 \mathrm{~nm}$ light with a pulse width of $10 \mathrm{~ns}$. Microcrystalline KDP was served as the standard. The particle sizes of the sieved sample and KDP were arranged from 25 to $260 \mu \mathrm{m}$ for the measurement of size-dependent SHG effect. The energy of each pulse was measured to be about $200 \mathrm{~mJ}$. The sample crystal was fixed on the bracket. An optical concave lens was used to adjust the diameter of the laser beam to obtain different intensities. The samples endured gradually enhanced radiation until their appearance changed under a microscope after the irradiation.

\subsection{Thermogravimetric Analysis}

The thermogravimetric analysis (TGA) was carried out on a SDTQ 600 simultaneous analyzer instrument (TA Instruments, New Castle, PA, USA). The crystal sample was added into an $\mathrm{Al}_{2} \mathrm{O}_{3}$ crucible and heated from room temperature to $800{ }^{\circ} \mathrm{C}$ at a heating rate of $10 \mathrm{~K} \mathrm{~min}^{-1}$ under flowing nitrogen gas.

\subsection{Theoretical Calculation}

The electronic structure and optical properties for the title compound were performed using the first principles plane-wave pseudopotential method implemented in the Cambridge Serial Total Energy Package (CASTEP) [38]. According to the experimental structure, the model was built and Rb atom was located at $(0.7500,0.3831,0.9670)$ in the unit cell because of its order. The optimized normal-conserving pseudopotentials [39] in the Kleinman-Bylander form are chosen and the valence electrons are $4 s, 4 p$ and $5 s$ for $\mathrm{Rb} ; 5 p, 5 d$ and $6 s$ electrons for $\mathrm{Hg}$; and $5 s, 5 p$ electrons for I. The local-density approximation (LDA) with a high kinetic energy cutoff of $900 \mathrm{eV}$ is adopted. Monkhorst-Pack [40] k point meshes with a density of $4 \times 4 \times 4$ points in the Brillouin zone of the unit cell are used. The scissor-factors-corrected LDA are used to calculate the second-order susceptibility $\chi^{2}$ based on the formula developed by Lin et al. [41]. 


\section{Conclusions}

A new ternary $\mathrm{RbHgI}_{3}$ with an orthorhombic space group Ama2 has been obtained by reaction of $\mathrm{HgI}_{2}$ and $\mathrm{RbI}(1: 1)$ in alcohol. The compound shows phase-matchable SHG of about seven times as strong as that of KDP, a wide transparent region (in the range of $0.48-25 \mu \mathrm{m}$ ), and a relatively good stability to the environment. Its crystals can be grown in solution. Owing to these properties, $\mathrm{RbHgI}_{3}$ appears to be a promising new NLO crystal applicable in the infrared region. Our future efforts will be devoted to growing large and high-quality crystals of $\mathrm{RbHgI}_{3}$ to further study its optical properties, for instance SHG coefficients, refractive indices and laser damage threshold.

Supplementary Materials: The following are available online at www.mdpi.com/2073-4352/7/5/148/s1: Figure S1: Figure S1. As grown crystal of $\mathrm{RbHgI}_{3}$; Figure S2: Figure S2. The calculated refractive indices for $\mathrm{RbHgI}_{3}$.

Acknowledgments: This work was supported by the National Science Foundation of China (No. 21301132 and No. 91022036) and Specialized Research Fund for the Doctoral Program of Higher Education of China (No. 20134219120002).

Author Contributions: Yanjun Li conceived and designed the experiments and wrote the paper; Yuxun Ding performed the experiments and analyzed the data; Yaming Li and Hongmin Liu carried out the characterization work; Xianggao Meng analyzed the crystal structure; Ye Cong, Jiang Zhang and Xuanke Li contributed reagents and materials; Jingui Qin, Xingguo Chen and Yanjun Li conceived and coordinated the project.

Conflicts of Interest: The authors declare no conflict of interest.

\section{References}

1. Burland, D.M.; Miller, R.D.; Walsh, A.C. Second-order nonlinearity in poled-polymer systems. Chem. Rev. 1994, 94, 31-75. [CrossRef]

2. Chai, B.H.T. CRC Handbook of Laser Science and Technology Supplement 2: Optical Materials; Weber, M.J., Ed.; CRC: Boca Raton, FL, USA, 1995.

3. Chen, C.T.; Wu, B.C.; Jiang, A.D.; You, M.G. A new-type ultraviolet SHG crystal- $\beta-\mathrm{BaB}_{2} \mathrm{O}_{4}$. Sci. Sin. Ser. B 1985, 28, 235-243.

4. Chen, C.T.; Wu, Y.C.; Jiang, A.D.; Wu, B.C.; You, G.M.; Li, R.K.; Lin, S.J. New nonlinear-optical crystal: $\mathrm{LiB}_{3} \mathrm{O}_{5}$. J. Opt. Soc. Am. B 1989, 6, 616-621. [CrossRef]

5. Chen, C.T.; Xu, Z.Y.; Deng, D.Q.; Zhang, J.; Wrong, G.K.L. The vacuum ultraviolet phase-matching characteristics of nonlinear optical $\mathrm{KBe}_{2} \mathrm{BO}_{3} \mathrm{~F}_{2}$ crystal. Appl. Phys. Lett. 1996, 28, 2930-2932. [CrossRef]

6. Chen, C.T.; Ge, N.; Lin, J.; Jiang, J.; Zeng, W.R.; Wu, B.C. Computer-Assisted Search for Nonlinear Optical Crystals. Adv. Mater. 1999, 11, 1071-1078. [CrossRef]

7. Chen, C.T.; Wang, G.L.; Wang, X.Y.; Xu, Z.Y. Deep-UV nonlinear optical crystal $\mathrm{KBe}_{2} \mathrm{BO}_{3} \mathrm{~F}_{2}$-discovery, growth, optical properties and applications. Appl. Phys. B 2009, 97, 9-25. [CrossRef]

8. Smith, W.L. KDP and ADP transmission in the vacuum ultraviolet. Appl. Opt. 1977, 16, 306-318. [CrossRef] [PubMed]

9. Kato, K. Parametric oscillation at $3.2 \mu \mathrm{m}$ in KTP pumped at $1.064 \mu \mathrm{m}$. IEEE J. Quantum Electron. 1991, 27, 1137-1140. [CrossRef]

10. Chemla, D.S.; Kupecek, P.J.; Robertson, D.S.; Smith, R.C. Silver thiogallate, a new material with potential for infrared devices. Opt. Commun. 1971, 3, 29-31. [CrossRef]

11. Boyd, G.D.; Kasper, E.H.M.; Mcfee, J.H.; Storz, F.G. Linear and nonlinear optical properties of AgGaS $\mathrm{A}_{2}$ $\mathrm{CuGaS}_{2}$, and $\mathrm{CuInS}_{2}$, and theory of the wedge technique for the measurement of nonlinear coefficients. IEEE J. Quantum Electron. 1972, 8, 563-573.

12. Boyd, G.D.; Buehler, E.; Storz, F.G. Linear and nonlinear optical properties of $\mathrm{ZnGeP}_{2}$ and CdSe. Appl. Phys. Lett. 1971, 18, 301-304. [CrossRef]

13. Liang, F.; Lin, L.K.Z.; Wu, Y.; Chen, C. Analysis and prediction of mid-IR nonlinear optical metal sulfides with diamond-like structures. Coordin. Chem. Rev. 2017, 333, 57. [CrossRef]

14. Guo, S.; Chi, Y.; Guo, G. Recent achievements on middle and far-infrared second-order nonlinear optical materials. Coord. Chem. Rev. 2017, 335, 44. [CrossRef] 
15. Jackson, A.G.; Ohmer, M.C.; LeClair, S.R. Relationship of the second order nonlinear optical coefficient to energy gap in inorganic non-centrosymmetric crystals. Infrared Phys. Technol. 1997, 38, 233-244. [CrossRef]

16. Chen, C.T.; Liu, G.Z. Recent Advances in Nonlinear Optical and Electro-Optical Materials. Annu. Rev. Mater. Sci. 1986, 16, 203-243. [CrossRef]

17. Zhang, G.; Li, Y.; Jiang, K.; Zeng, H.; Liu, T.; Chen, X.; Qin, J.; Lin, Z.; Fu, P.; Wu, Y.; et al. A New Mixed Halide, $\mathrm{Cs}_{2} \mathrm{HgI}_{2} \mathrm{Cl}_{2}$ : Molecular Engineering for a New Nonlinear Optical Material in the Infrared Region. J. Am. Chem. Soc. 2012, 134, 14818-14822. [CrossRef] [PubMed]

18. Zhang, G.; Qin, J.; Liu, T.; Fu, P.; Wu, Y.; Chen, C. Synthesis, Characterization, and Crystal Growth of $\mathrm{Cs}_{2} \mathrm{Hg}_{3} \mathrm{I}_{8}$ : A New Second-Order Nonlinear Optical Material. Cryst. Growth Des. 2008, 8, 2946-2949. [CrossRef]

19. Wu, Q.; Li, Y.; Chen, H.; Jiang, K.; Li, H.; Zhong, C.; Chen, X.; Qin, J. HgBrI: A promising nonlinear optical material in IR region. Inorg. Chem. Commun. 2013, 34, 1-3. [CrossRef]

20. Liu, T.; Qin, J.; Zhang, G.; Zhu, T.; Niu, F.; Wu, Y.; Chen, C. Mercury Bromide $\left(\mathrm{HgBr}_{2}\right)$ : A promising nonlinear optical material in IR region with a high laser damage threshold. Appl. Phys. Lett. 2008, 93, 091102. [CrossRef]

21. Li, Y.; Wang, M.; Zhu, T.; Meng, X.; Zhong, C.; Chen, X.; Qin, J. Synthesis, crystal structure and properties of a new candidate for nonlinear optical material in the IR region: $\mathrm{Hg}_{2} \mathrm{BrI}_{3}$. Dalton Trans. 2012, 41, 763-766. [CrossRef] [PubMed]

22. Lv, S.W.; Wu, Q.; Meng, X.; Kang, L.; Zhong, C.; Lin, Z.; Hu, Z.; Chen, X.; Qin, J. A promising new nonlinear optical crystal with high laser damage threshold for application in the IR region: Synthesis, crystal structure and properties of noncentrosymmetric $\mathrm{CsHgBr}_{3}$. J. Mater. Chem. C. 2014, 2, 6796-6801. [CrossRef]

23. Dang, Y.; Meng, X.; Jiang, K.; Zhong, C.; Chen, X.; Qin, J. A promising nonlinear optical material in the Mid-IR region: New results on synthesis, crystal structure and properties of noncentrosymmetric $\beta-\mathrm{HgBrCl}$. Dalton Trans. 2013, 42, 9893-9897. [CrossRef] [PubMed]

24. Zhang, G.; Qin, J.; Liu, T.; Li, Y.; Wu, Y.; Chen, C. $\mathrm{NaSb}_{3} \mathrm{~F}_{10}$ : A new second-order nonlinear optical crystal to be used in the IR region with very high laser damage threshold. Appl. Phys. Lett. 2009, 95, 1. [CrossRef]

25. Wu, Q.; Meng, X.; Zhong, C.; Chen, X.; Qin, J. $\mathrm{Rb}_{2} \mathrm{CdBr}_{2} \mathrm{I}_{2}$ : A new IR nonlinear optical material with a large laser damage threshold. J. Am. Chem. Soc. 2014, 136, 5683-5686. [CrossRef] [PubMed]

26. Holmes, D.E.; Harvill, M.L.; Bogan, L.D. A survey of $\mathrm{ABX}_{3}$ halides for optical second harmonic generation. Mat. Res. Bull. 1975, 10, 753-759. [CrossRef]

27. George, A.J.; Marcus, V. Crystal structures of the red, yellow, and orange forms of mercuric iodide. Inorg. Chem. 1967, 6, 396-399.

28. Cortona, P. Direct determination of self-consistent total energies and charge densities of solids: A study of the cohesive properties of the alkali halides. Phys. Rev. B 1992, 46, 2008. [CrossRef]

29. Avdienko, K.I.; Badikov, D.V.; Badikov, V.V.; Chizhikov, V.I.; Panyutin, V.L.; Shevyrdyaeva, G.S.; Scherbakov, S.I.; Scherbakova, E.S. Optical properties of thallium mercury iodide. Opt. Mater. 2003, 23, 569-573. [CrossRef]

30. Sathiskumar, S.; Kathiravan, P.; Balakrishnan, T. Cationic Coordination Compound $\mathrm{Cs}_{2} \mathrm{Hg}_{3} \mathrm{I}_{8}$ for IR NLO Material: Synthesis, Crystal Growth and Characterizations. In Proceedings of the AIP conference Proceedings 1665, Tamilnadu, India, 16-20 December 2014; p. 2814.

31. Kurtz, S.K.; Perry, T.T. A Powder Technique for the Evaluation of Nonlinear Optical Materials. J. Appl. Phys. 1968, 39, 3798-3813. [CrossRef]

32. Nikogosyan, D.N. Nonlinear Optical Crystals: A Complete Survey; Springer: New York, NY, USA, 2005; pp. 102-103.

33. Godby, R.W.; Schlüter, M.; Sham, L.J. Trends in self-energy operators and their corresponding exchange-correlation potentials. Phys. Rev. B 1987, 36, 6497. [CrossRef]

34. Sheldrick, G.M. SHELXTL, Version 6.14; Bruker Analytical x-ray Instruments, Inc.: Madison, WI, USA, 2003.

35. Sheldrick, G.M. SHELXS-97-A Program for Automatic Solution of Crystal Structures; University of Goettingen: Goettingen, Germany, 1997.

36. Sheldrick, G.M. SHELXL-97-A Program for Crystal Structure Refinement; University of Goettingen: Goettingen, Germany, 1997.

37. Wendlandt, W.H.; Hecht, H.G. Reflectance Spectroscopy; Interscience: New York, NY, USA, 1966; pp. 62-65.

38. Clark, S.J.; Segall, M.D.; Pickard, C.J.; Hasnip, P.J.; Probert, M.I.J.; Refson, K.; Payne, M.C. First principles methods using CASTEP. Z. Kristallogr. 2005, 220, 567-570. [CrossRef] 
39. Lin, J.S.; Qtseish, A.; Payne, M.C.; Heine, V. Optimized and transferable nonlocal separable ab initio pseudopotentials. Phys. Rev. B 1993, 47, 4174. [CrossRef]

40. Perdew, J.P.; Burke, K.; Ernzerhof, M. Generalized Gradient Approximation Made Simple. Phys. Rev. Lett. 1996, 77, 3865. [CrossRef] [PubMed]

41. Lin, J.; Lee, M.; Lin, Z.; Chen, C.; Pickard, C. Mechanism for linear and nonlinear optical effects in beta-BaB $\mathrm{O}_{4}$ crystals. Phys. Rev. B Condens. Matter Mater. Phys. 1999, 60, 13380. [CrossRef] 